\title{
Error Probabilities of Coherent Optical Heterodyne FSK-CDMA Signals in a Noncoherent Detection System Degraded by Laser Phase Noise
}

\author{
Kent C. M. Varnum \\ Naval Ordnance Missile Test Station \\ White Sands Missile Range \\ NM 88002
}

\author{
R. Clark Robertson \\ Electrical and Computer Eng. Dept. \\ Naval Postgraduate School \\ Monterey, CA 93943
}

\begin{abstract}
An error probability analysis is performed for noncoherent detection of optical heterodyne frequency-shift keying (FSK) direct sequence spread-spectrum codedivision multiple access (CDMA) signals corrupted by laser phase noise, receiver noise, and multiuser noise. Receiver noise is included in the analysis as an additive white Gaussian process. The multiuser noise is also modeled as a Gaussian process.

The application of spread spectrum techniques to an optical channel can increase system simultaneous user capacity significantly with minimal performance degradation for long spreading codes. An FSK-CDMA system with low, moderate, and high user bit rates as compared with the laser linewidth is analyzed over different spreading code lengths for increasing numbers of users. The low bit rate system performs poorly even when the spreading code is long and the number of simultaneous users is very small. On the other hand, a high bit rate system which uses a spreading code with $2^{11}$ chips can support up to seventy-five users if the maximum allowed probability of bit error is $10^{-6}$.
\end{abstract}

\section{Introduction}

Optical systems are degraded by standard receiver noise, shot noise in the photodetector, and phase noise in the transmitting laser. The impact of receiver and photodetector shot noise is significantly reduced by the application of optical heterodyne techniques which are very similar to standard RF heterodyne techniques. Unlike direct detection systems, optical heterodyne systems mix a locally generated lightwave with the received signal which is then detected by a photodetector. The resulting electrical signal is a replica of the optical signal translated down in frequency, usually to the microwave frequency range. Mixing the incoming optical signal with a local laser provides strong optical input power to the photodetector. The strong local laser condition drastically reduces the effect of the receiver thermal noise and photodetector shot noise. Unfortunately, the addition of a local laser at the receiver increases the effect of the laser phase noise on system performance. Laser phase noise is a noise mechanism inherent to the physical na- ture of all lasers that impresses random phase and amplitude modulation on the otherwise monochromatic laser output. In optical heterodyne systems, the laser phase noise of the transmit and receive lasers is additive. Current research indicates that in order to attain reasonable bit error performance, the system filter bandwidth must be at least ten times the sum of the laser phase noise bandwidths of the transmitting and local lasers [1]. Current semi-conductor lasers may have a laser phase noise linewidth of up to 50 $\mathrm{MHz}$ and require a channel bandwidth of up to 100 $\mathrm{MHz}$. For user bit rates less than or equal to the laser phase noise bandwidth, the channel spacing required in wavelength division multiplexing (WDM) systems to ensure sufficient guardbands results in an extremely inefficient use of available bandwidth.

Future systems will have to accommodate more users with higher bit rates. In this paper we address the high bit rate systems that will be required by future users. As a means of improving the multiuser capacity of high bit rate optical communications systems, we propose the implementation of codedivision multiple access (CDMA) techniques in an optical heterodyne FSK system with noncoherent detection. CDMA is a type of spread-spectrum that adds multiuser capability by spreading and despreading each user data signal with a unique digital code. Each system user is assigned a particular code sequence which is used to encode each data bit. In this paper we model the spreading codes with random codes. Random codes are constructed of a sequence of random variables taking values $\{+1,-1\}$ with equal probability, and the sequences assigned to different users are mutually independent [2]. The application of CDMA techniques improves standard optical heterodyne WDM system performance by increasing user capacity on a given WDM channel with minimal impact on system performance.

To illustrate the improvement realized by the application of CDMA techniques this paper analyzes a multiuser optical heterodyne FSK-CDMA system. System performance is measured by the probability of bit error as a function of the combined system laser linewidth, bit time product, and the number of simul- 
taneous users. Both receiver noise and multiuser noise are modeled as additive white Gaussian noise. The ratio of signal power to the noise power from standard receiver noise and shot noise is assumed to be large enough so that the primary degradation in performance is due to laser phase noise and multiuser noise. It is assumed that the user bit stream consists of a mutually independent random series of 'ones' and 'zeros'. The system will only transmit a signal when the user has data to send, otherwise the station will remain idle. Each transmitter FSK modulates a semiconductor laser diode with the user bit stream. It is assumed that there is negligible interference between the two FSK tones. After the user bit stream FSK modulates the transmit laser, the transmitter encodes the FSK bit stream into the spreading sequence through binary phase-shift keying producing a high frequency chip stream consisting of two frequencies, each phase modulated with the user code sequence. The transmitted signal is then optically mixed with other transmitter signals in the common optical fiber channel. The system under consideration is also considered to be a 'power balanced network'. In this type of network every signal, desired or undesired, is transmitted with the same power.

At the receiver, the optical signal is down converted to an intermediate (IF) frequency with a locally generated optical signal and detected with a photodector. The remainder of the receiver consists of two separate receivers, called branches, which demodulate the electrical IF signal at the photodector output. One branch is matched to $f_{1}$ and the other is matched to $f_{0}$, where $f_{0}$ and $f_{1}$ are the IF frequencies corresponding to a bit 'zero' and a bit 'one', respectively. Each of the frequency matched branches consists of two sub-branches in quadrature. Each sub-branch of the receiver adds a locally generated IF signal to the incoming composite IF signal. The locally generated IF signal added to the incoming signal is phase modulated with the user code sequence. The resulting electrical signal is then integrated over the bit interval and noncoherently demodulated by a square-law detector. The output of each square-law detector is then added to its quadrature component to form the correlation statistics. The correlation statistics produced by each of the two frequency matched branches are then input to a comparator for bit recovery. As with standard FSK systems, the comparator is a threshold device with the threshold set at zero.

\section{Analysis}

The performance of the demodulator depends on the statistics of the output waveform $\mu_{i}(T), i=0,1$ of each branch or the receiver corrupted by laser phase noise, receiver shot noise, and multiuser noise. The probability of bit error is

$$
P_{b}=0.5\left[P_{0}+P_{1}\right]
$$

where equally likely signalling is assumed and

$$
P_{0}=\operatorname{Pr}\left\{\mu_{1}>\mu_{0} \mid 0\right\}
$$

is the probability of making an error when a 'zero' is sent and

$$
P_{1}=\operatorname{Pr}\left\{\mu_{0}>\mu_{1} \mid 1\right\}
$$

is the probability of making an error when a 'one' is sent.

\subsection{Derivation of the Conditional Proba- bility of Bit Error}

The IF signal received by an individual user during each $T_{b}$ interval is expressed

$$
s(t)=\operatorname{Re}\left\{r(t) \exp \left(j \omega_{i} t\right)\right\}
$$

where $i=1,0$ and

$$
r(t)=a(t) S \exp [j \theta(t)]+n(t)
$$

is the complex baseband signal where the power received in the optical pulse is $S^{2} / 2, \theta(t)$ is the composite phase noise due to both the transmitting and receiving lasers, and $n(t)$ is the complex noise due to the combination of additive white Gaussian receiver noise and multiuser noise. The user code sequence is represented by a periodic sequence of unit amplitude rectangular pulses $a(t)=\{-1,+1\}$ of duration $T_{c}$ where $T_{c}=T_{b} / N$ and $N$ is the code length.

Due to the symmetry of the receiver, the probability of making an error is the same for both a transmitted 'one' and transmitted 'zero'; that is, $P_{1}=P_{0}$ Because of this symmetry, it is only necessary to examine receiver performance for either a transmitted 'one' or a transmitted 'zero'. The signal is, therefore, assumed to be present in the branch of the receiver corresponding to user bit 'one'. Then for the receiver branch matched to frequency $f_{1}$, the output of the ideal baseband integrators, each with an integration time $T_{b}$, is

$$
\zeta_{1 i}\left(T_{b}\right)=\operatorname{Re}\left[\zeta_{1}\left(T_{b}\right)\right]
$$

for the in-phase channel and

$$
\zeta_{1 q}\left(T_{b}\right)=\operatorname{Im}\left[\zeta_{1}\left(T_{b}\right)\right]
$$

for the quadrature channel where

$$
\zeta_{1}\left(T_{b}\right)=\frac{S}{T_{b}} \int_{0}^{T_{b}} \exp [j \theta(t)] d t+\hat{n}
$$

and $\hat{n}=\hat{n}_{i}+j \hat{n}_{q}$ is a zero mean, additive white Gaussian complex baseband noise sample which includes both receiver noise and multiuser noise. For the receiver branch matched to frequency $f_{0}$, the output of the in-phase and quadrature baseband integrators during each $T_{b}$ interval is $\hat{n}_{i}$ and $\hat{n}_{q}$, respectively.

These signals are detected by ideal square-law detectors and the overall decision statistic for each branch is obtained by summing the square-law detector outputs for the in-phase and quadrature channels. For a given of the decision statistic $\mu_{1}$ for branch 1 , the conditional error probability is

$$
P_{1}\left(\mu_{1}\right)=\int_{\mu_{1}}^{\infty} p\left(\mu_{0} \mid 1\right) d \mu_{0}
$$


where

$$
p\left(\mu_{0} \mid 1\right)=\frac{1}{\sigma^{2}} \exp \left(-\frac{\mu_{0}}{\sigma^{2}}\right)
$$

is the probability density function (pdf) for $\mu_{0}$ when a data bit 'one' is sent and $\sigma^{2}$ is the combined noise power of the in-phase and quadrature square-law detector outputs. The average error probability conditional on the laser phase noise is obtained by averaging over all values of $\mu_{1}$ to get

$$
P_{b \mid X}(x)=\int_{0}^{\infty} P_{1}\left(\mu_{1}\right) p\left(\mu_{1} \mid 1, x\right) d \mu_{1}
$$

where $X$ represents the magnitude of the random variable determined by the laser phase noise and

$$
p\left(\mu_{1} \mid 1, x\right)=\frac{1}{\sigma^{2}} \exp \left(-\frac{\mu_{1}+S^{2} x^{2}}{\sigma^{2}}\right) I_{0}\left(\frac{2 S x \sqrt{\mu_{1}}}{\sigma^{2}}\right)
$$

is the pdf for $\mu_{1}$ conditioned by the laser phase noise when a data bit 'one' is sent and $I_{0}(\bullet)$ represents the modified Bessel function of zero order. The unconditional probability of bit error is thus

$$
P_{b}=\int_{0}^{1}\left[\int_{0}^{\infty} p\left(\mu_{1} \mid 1, x\right) P_{1}\left(\mu_{1}\right) d \mu_{1}\right] p_{X}(x) d x
$$

where $p_{X}(x)$ is the pdf for the random variable $X$. The expression for the probability of bit error is now be simplified.

Combining (9)-(12), we get

$$
P_{b}=\frac{1}{2} \int_{0}^{1} \exp \left(-\frac{S^{2} x^{2}}{2 \sigma^{2}}\right) p_{X}(x) d x
$$

which must be evaluated numerically. If the effect of laser phase noise is negligible, then $p_{X}(x) \approx \delta(x-$ 1) where $\delta(\bullet)$ is the Dirac delta function, and (14) reduces to

$$
P_{b}=\frac{1}{2} \exp \left(-\frac{S^{2}}{2 \sigma^{2}}\right)
$$

which is the probability of bit error for a conventional FSK receiver with noncoherent detection.

\subsection{Analytic Expressions for the Noise Terms}

The major source of degradation to an optical fiber communication system is laser phase noise. In optical heterodyne systems, both the transmit and local lasers add laser phase noise to the received signal. This causes the introduction of a random frequency deviation to the IF signal related to the sum of the linewidths of both lasers. Due to the filter response of the initial IF filter in each branch of the FSK-CDMA receiver, the pdf of the random variable $X$ determined by the laser phase noise depends strictly upon the laser linewidth $\beta$ and the bit rate $T_{b}$. Direct evaluation of this pdf is computationally intensive since $\theta(t)$ in $(8)$ is a Brownian motion process. The pdf of $X$ can be evaluated through numerical integration and Monte Carlo simulation; however, large amounts of computational time are required [3]. Attempts to simplify the analysis of the probability of bit error in lightwave systems corrupted by laser phase noise through the derivation of a closed form analytical model representing $p_{\boldsymbol{X}}(x)$ results in a curve fit approximation of the actual pdf. For an integrate and dump filter the pdf of the random variable $X$ determined by the laser phase noise is approximated as [4]

$$
p_{X}(x)=\alpha[1-\exp (-\alpha)]^{-1} \exp [-\alpha(1-x)]
$$

for $0 \leq x \leq 1$ and zero otherwise where

$$
\alpha=\frac{1.6}{\beta T_{b}}\left(1+0.5 \sqrt{\beta T_{b}}\right)
$$

The accuracy of this model has been demonstrated over many different system signalling rates [3]. We use this expression to represent the random variable $X$ determined by the laser phase noise.

Spread-spectrum code-division multiple access (CDMA) is an asynchronous multiple access communication scheme in which many users share a common bandwidth. In CDMA each user is assigned a particular code sequence which is used to modulate the carrier depending on the digital data [2]. Under ideal conditions, each particular user code is orthogonal to every other user code, and as a result, invisible to other users. This is not the case in practical systems. A particular user recovers his coded bit stream through a receiver matched to the particular user's code. Other simultaneous user's signals will corrupt the received signal and appear as noise in the particular user's receiver. In many cases of interest, the multiuser noise is represented as a Gaussian random process. The validity of the Gaussian multiuser noise model degrades at lower code lengths and fewer numbers of users, but for small numbers of users the laser phase noise dominates system performance. In addition, system performance is less critical when the number of users is small.

In this paper we assume the use of random signature sequences. Random signature sequences are constructed of a sequence of random variables taking values $\{+1,-1\}$ with equal probability, and all sequences are mutually independent. Analysis using random signature sequences is mathematically simpler.

Much work has been done in recent years to characterize the statistics of direct-sequence spreadspectrum codes. The difficulty in analyzing such systems is the fact that they are asynchronous and proper analysis requires characterizing not only the periodic but also the aperiodic cross-correlation properties. Most current models treat phase shifts, time delays, and data symbols as mutually independent random variables. The Gaussian random variable that describes the multiuser noise for random codes is zero mean with a variance $[2,6]$

$$
\sigma^{2}=\frac{S^{2}(K-1)}{3 N}+\frac{2 N_{0}^{s}}{T_{b}}
$$

where $N_{0}^{s} / 2$ is the two-sided spectral density of the additive white Gaussian receiver noise. 


\section{Numerical Results}

The computation of the probability of bit error involves a numerical evaluation of (14) for different lengths of random user signature sequences over the range of simultaneous users the system can support. The ratio of signal power to the noise power due to receiver shot noise (SNR) is taken to be $16 \mathrm{~dB}$. This yields a probability of bit error of $10^{-9}$ when laser phase noise and multiuser noise are negligible. It is also assumed that the optical signal power of an individual user is normalized to unity and that the transmitter equally balances the active user signals within the composite optical signal. The number of chips in the random user code is varied from $2^{8}$ to $2^{11}$. The three cases of a relatively small bit rate, a moderately high bit rate, and a very high bit rate, corresponding to laser linewidth, bit time products of $\beta T_{b}=0.3,0.1$, and 0.02 , respectively, are investigated. The resulting system performance is shown in Figs. 1-3.

As can be seen in Figs. 1-3, for an SNR of $16 \mathrm{~dB}$ the high bit rate is required in order to obtain a probability of bit error of $10^{-6}$ or less. Regardless of system bit rate, the probability of bit error increases rapidly with the addition of just a few users for short code lengths. Long code lengths are required in order to support more than just a few simultaneous users without a significant performance degradation. For a bit rate ten times the laser linewidth, a ratio currently a rule of thumb for optical communication systems, the minimum probability of bit error is still significantly larger than that obtained with a high bit rate system. As can be seen by comparing Fig. 2 with Fig. 3 , a bit rate fifty times the laser linewidth, five times greater than the customary rule of thumb, is required to eliminate the negative effects of laser phase noise. The results also exhibit the standard CDMA characteristics in that they are fairly steep for low number of users and flat at high usage levels [5]. This behavior is demonstrated for all system bit rates examined (and, thus, for varying effects of laser phase noise on system performance). The steepness of the slope for low numbers of users is more pronounced for the high bit rate system; nevertheless, the high bit rate system is still markedly superior to the system with $\beta T_{b}=0.1$. For example, for a user code with $2^{11}$ chips and a maximum probability of bit error of $10^{-6}$, the high bit rate system can support up to seventy-five simultaneous users, while the minimum probability of bit error for the system with $\beta T_{b}=0.1$ is greater than $10^{-6}$ for even a single user due to the effects of laser phase noise. For a user code with $2^{9}$ chips and a maximum probability of bit error of $10^{-5}$, the high bit rate system can support up to thirty simultaneous users, while the system with $\beta T_{b}=0.1$ can support only about twelve simultaneous users. Due to the limitations on system performance imposed by laser phase noise, the minimum probability of bit error for the low bit rate system is greater than $10^{-3}$ regardless of the number of simultaneous users and code length.

The effect of laser phase noise on system performance when only one user is transmitting (i.e., there is no multiuser noise) is illustrated in Fig. 4. As can be seen, even a significant increase in signal energy will not substantially improve system performance for systems with a low bit rate. As noted earlier, for a bit rate ten times the laser linewidth, system performance is still significantly poorer than when laser phase noise is absent; although, as already demonstrated, a probability of bit error of less than $10^{-6}$ is possible with reasonable values of SNR. A bit rate fifty times the laser linewidth is required in order to virtually eliminate the negative effects of laser phase noise if a minimum probability of bit error of $10^{-9}$ is required; however, virtually identical performance can be obtained at the expense of about $1 \mathrm{~dB}$ of signal energy by relaxing the bit rate requirement from fifty to to twenty times the laser linewidth.

\section{Conclusions}

To improve overall optical communications system performance, we propose the application of CDMA spread spectrum techniques to a standard optical heterodyne FSK system employing Wavelength Division Multiplexing (WDM) to obtain multiple simultaneous user capabilities. This system can greatly improve system user capacity without a substantial increase in the probability of bit error provided the bit rate is high enough and the code is long enough.

\section{References}

[1] J. Salz, 'Coherent Lightwave Communications,' AT \& T Technical Journal, vol. 64, pp. 2153-2207, Dec. 1985 .

[2] M. B. Pursley, 'Performance Evaluation for Phase-Coded Spread-Spectrum Multiple-Access Communication-Part I: System Analysis,' IEEE Trans. on Communications, vol. COM-25, pp. 795799, Aug. 1977.

[3] G. J. Foschini, L. J. Greenstein, and G. Vannucci, 'Noncoherent Detection of Coherent Lightwave Signals Corrupted by Phase Noise, IEEE Trans. on Communications, vol. 36, pp. 306-314, Mar. 1988.

[4] G. J. Foschini, G. Vannucci and L. J. Greenstein, 'Envelope Statistics for Filtered Optical Signals Corrupted by Phase Noise,' IEEE Trans. on Communications, vol. 37, pp. 1293-1302, Dec. 1989.

[5] G. L. Turin, 'The Effects of Multipath and Fading on the Performance of Direct-Sequence CDMA Systems,' IEEE J. on Selected Areas in Communications, vol. SAC-2, pp. 597-603, Jul. 1984.

[6] E. A. Geraniotis, 'Performance of Noncoherent Direct-Sequence Spread-Spectrum MultipleAccess Communications,' IEEE J. on Selected Areas In Communications, vol. SAC-3, pp. 687-694, Sept. 1985. 


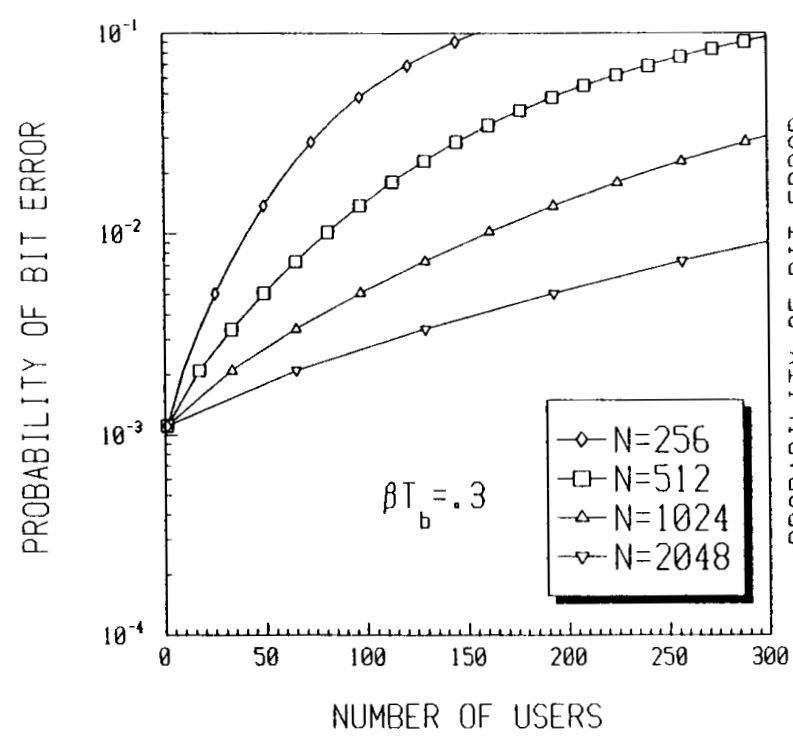

Figure 1: Probability of bit error for high order code lengths and low bit rate $\left(\beta T_{b}=0.3\right)$ with $S N R=16$ dB.

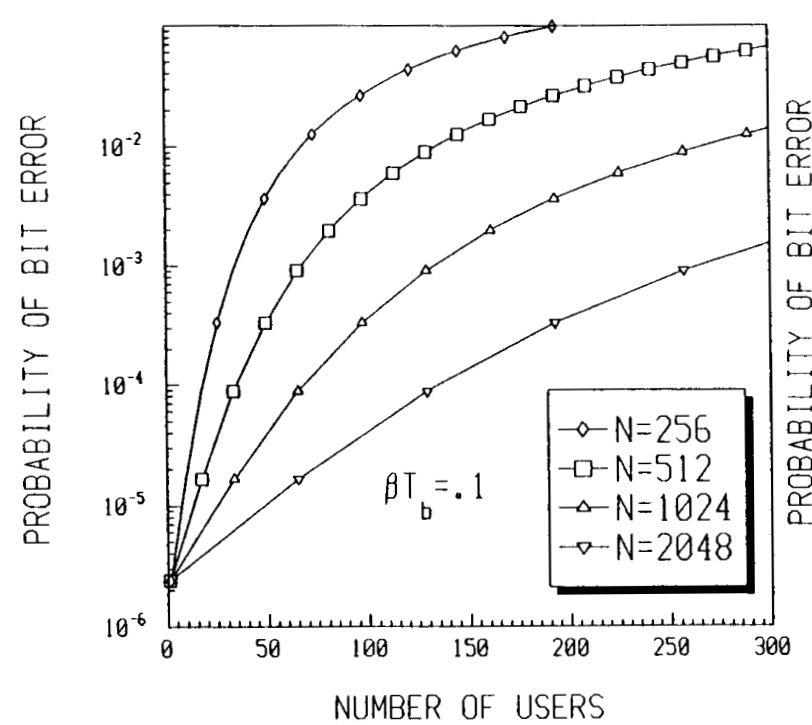

Figure 2: Probability of bit error for high order code lengths and moderate bit rate $\left(\beta T_{b}=0.1\right)$ with SNR $=16 \mathrm{~dB}$.

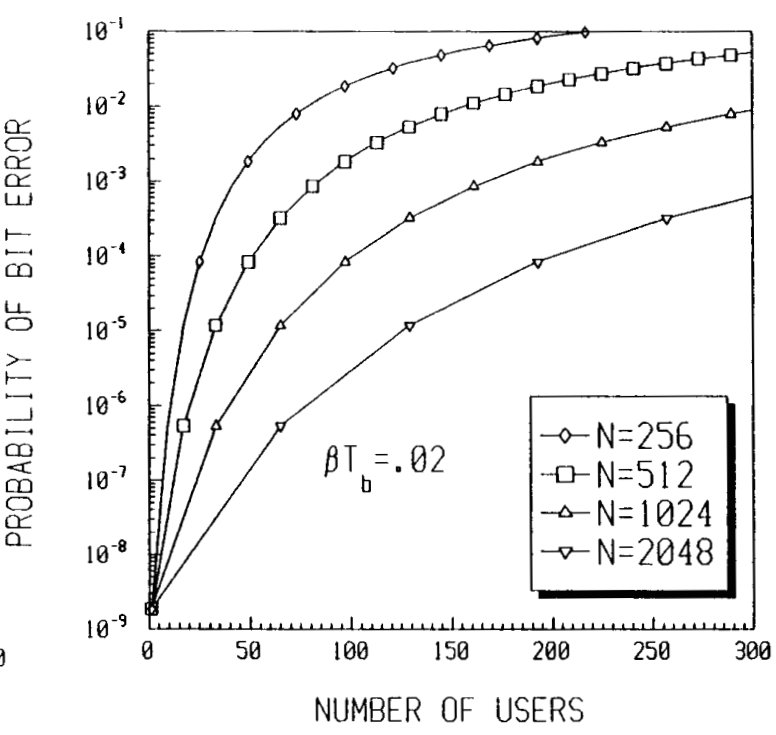

Figure 3: Probability of bit error for high order code lengths and high bit rate $\left(\beta T_{b}=0.02\right)$ with $\mathrm{SNR}=16$ dB.

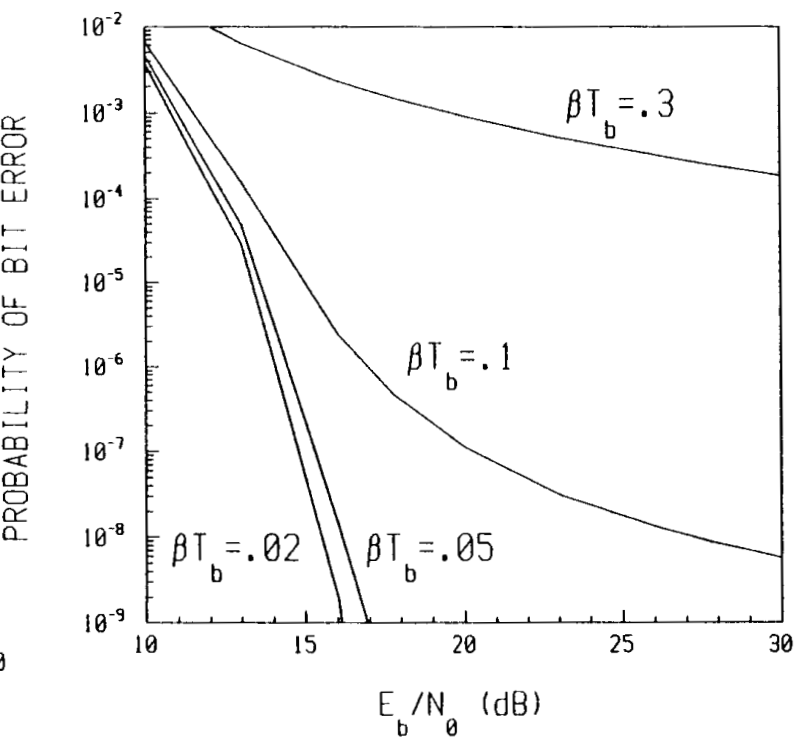

Figure 4: System performance for a single user with $\beta T_{b}$ as a parameter. 